\title{
LA-UR-21-21679
}

Approved for public release; distribution is unlimited.

Title: $\quad$ Proliferation Monitoring with Hidden Markov Models

Author(s): $\quad$ Hollis, Andrew

Tompkins, George

Wilson, Alyson

Smith, Ralph

Intended for: $\quad$ Next-Gen Al for Proliferation Detection: Domain Aware Methods (Virtual Workshop)

Issued: $\quad$ 2021-02-22 
Disclaimer:

Los Alamos National Laboratory, an affirmative action/equal opportunity employer, is operated by Triad National Security, LLC for the National Nuclear Security Administration of U.S. Department of Energy under contract 89233218CNA000001. By approving this article, the publisher recognizes that the U.S. Government retains nonexclusive, royalty-free license to publish or reproduce the published form of this contribution, or to allow others to do so, for U.S. Government purposes. Los Alamos National Laboratory requests that the publisher identify this article as work performed under the auspices of the U.S. Department of Energy. Los Alamos National Laboratory strongly supports academic freedom and a researcher's right to publish; as an institution, however, the Laboratory does not endorse the viewpoint of a publication or guarantee its technical correctness. 


\section{Proliferation Monitoring with Hidden Markov Models}

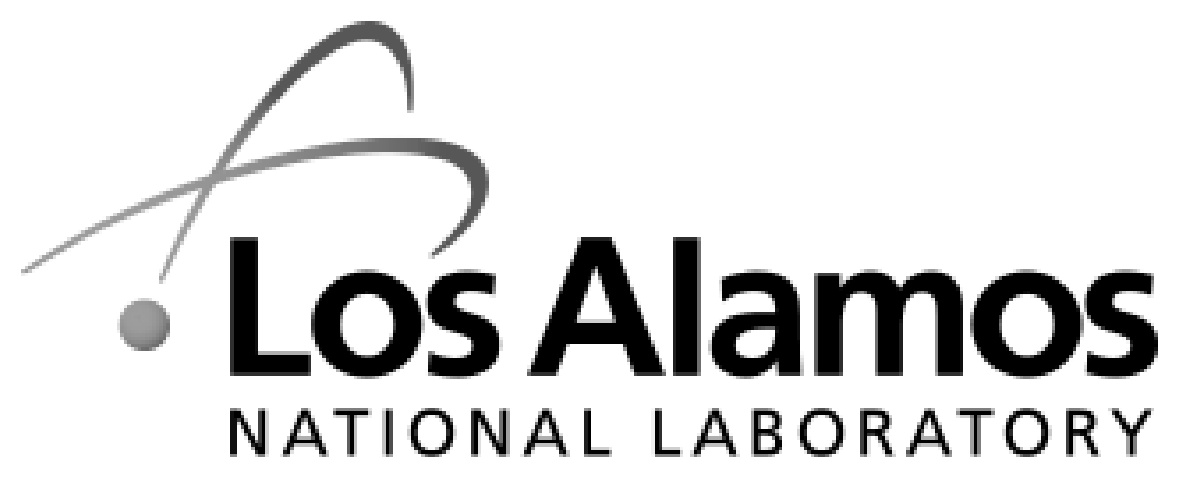

EST. 1943
Andrew Hollis, George Tompkins, Alyson Wilson, and Ralph Smith January, 2021 


\section{Proliferation Monitoring}

- Non-proliferation goal: monitor manufacturing and testing processes that might present a proliferation risk.

- Problem: data collected from monitoring systems does not yield direct knowledge of the activity underway.

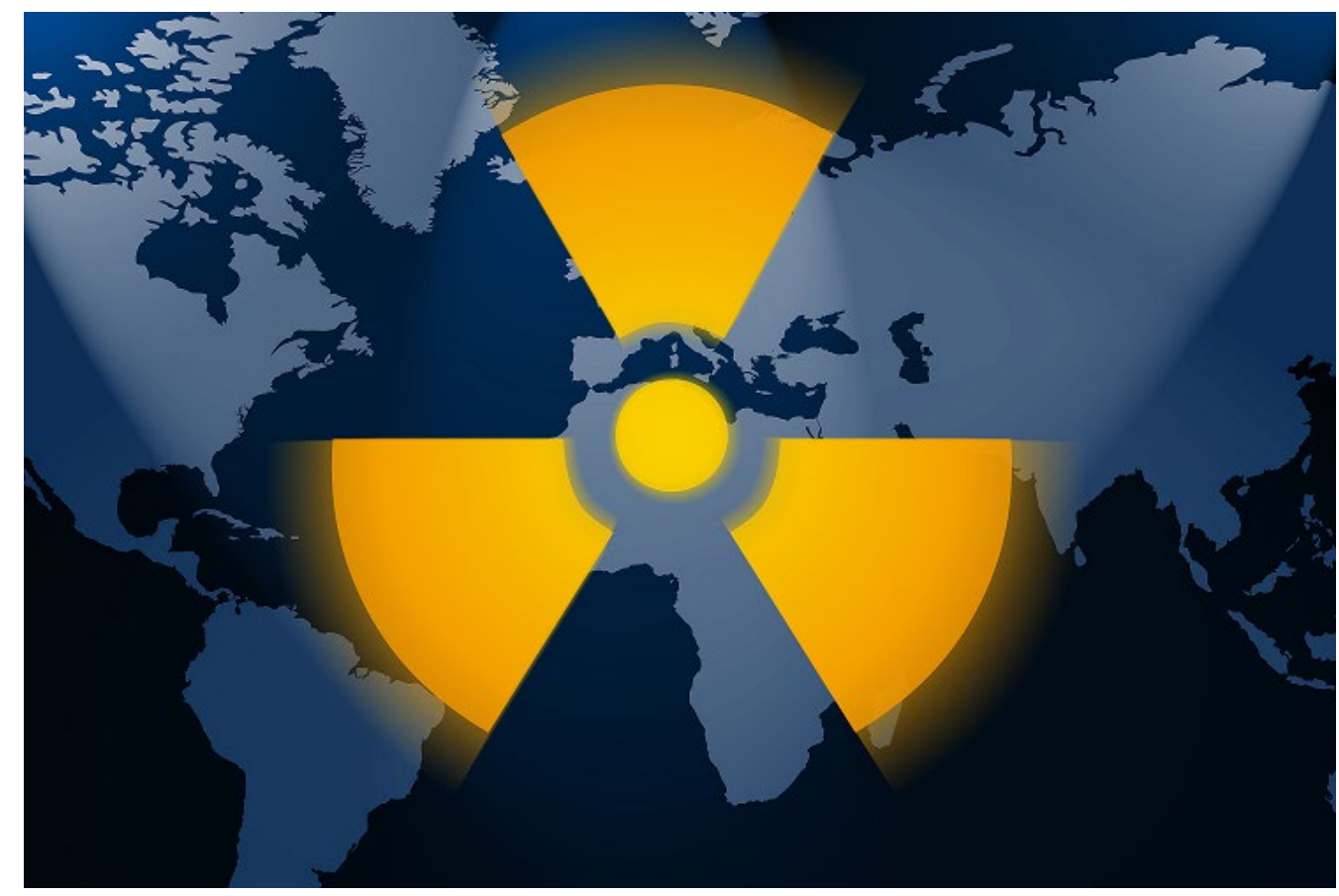




\section{Proliferation Monitoring Challenges}

- Goal: develop a statistical model that combines

- data observed from the process

- domain knowledge about the process

- This model should describe

- process of interest (unobserved)

- process data (observed)

- relationship between the process and the data
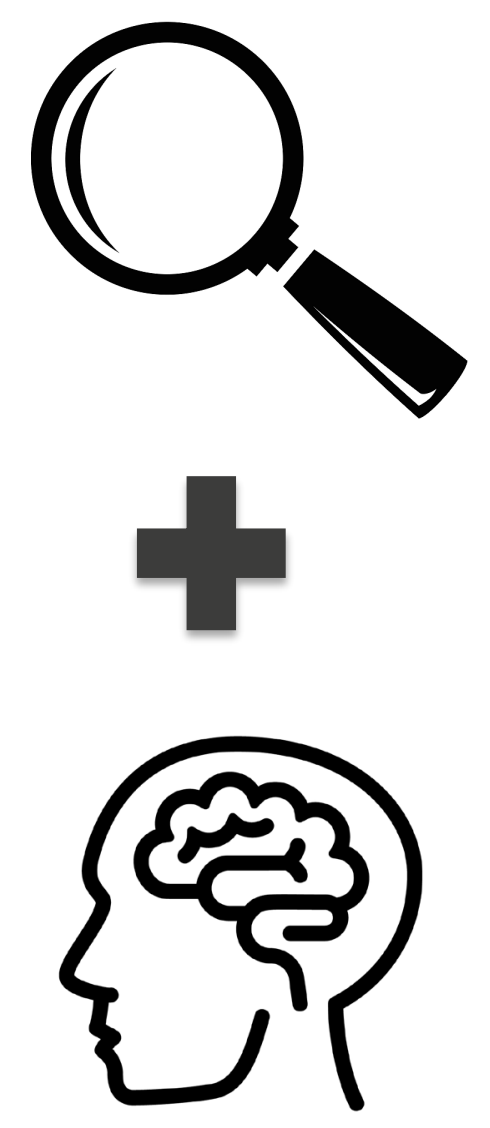


\section{Simple HMM Example}

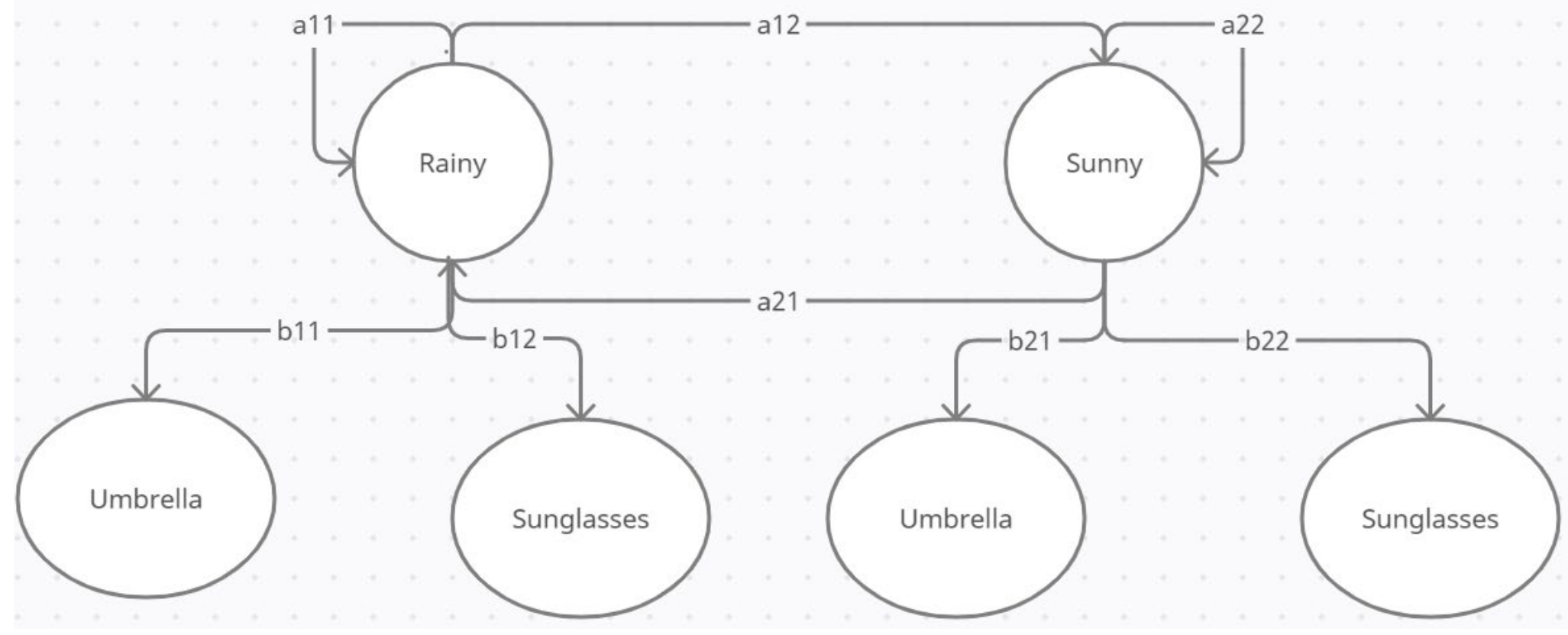

Simple Weather HMM 


\section{HMM Data Stream}

\section{Unobserved Process}

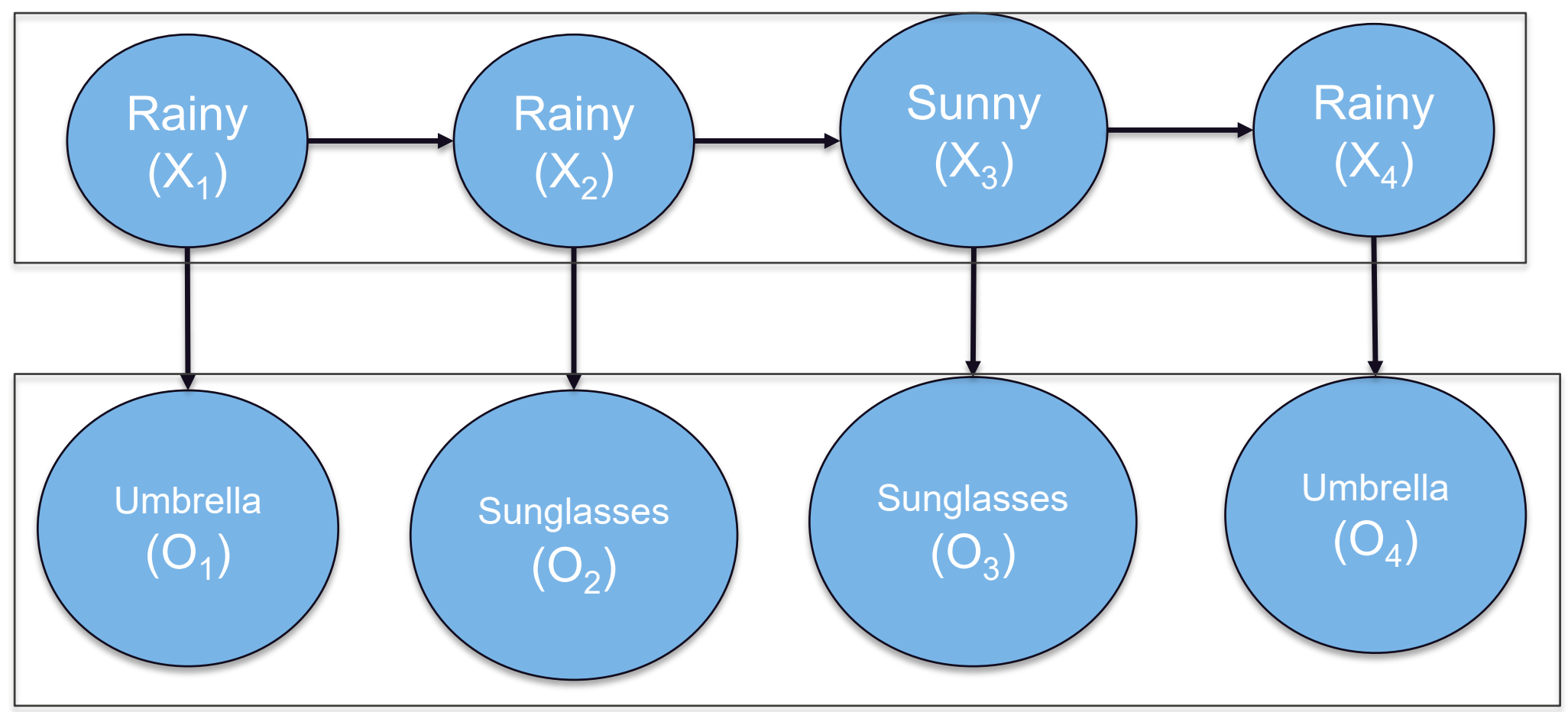

Observation Sequence 


\section{HMM Parameters}

- Initial State Probabilities: $\pi=\left(\pi_{1}, \ldots, \pi_{N}\right), \pi_{i}=P\left(X_{1}=i\right)$

- Observation Probabilities: $B=\left(\begin{array}{ccc}b_{11} & \cdots & b_{1 p} \\ \vdots & \ddots & \vdots \\ b_{N 1} & \cdots & b_{N p}\end{array}\right), b_{i j}=P\left(O_{t}=j \mid X_{t}=i\right)$, $i=1, \ldots, N, j=1, \ldots, p$, and $t=1, \ldots, n$.

- Transition Probabilities: $A=\left(\begin{array}{ccc}a_{11} & \cdots & a_{1 N} \\ \vdots & \ddots & \vdots \\ a_{N 1} & \cdots & a_{N N}\end{array}\right), a_{i j}=P\left(X_{t}=j \mid X_{t-1}=i\right)$, $i, j=1, \ldots, N$, and $t=1, \ldots, n$. 


\section{HMM Inference}

- The HMM can be used to compute

\section{State Distribution at $\mathrm{t}=3$}

$$
\gamma_{t}(i)=\mathrm{P}\left(X_{t}=i \mid \boldsymbol{O}, \boldsymbol{\lambda}\right),
$$

where $\lambda=(A, B, \boldsymbol{\pi})$ and $\boldsymbol{O}=$ $\left(O_{1}, \ldots, O_{n}\right)$.

- Takeaway: Infer most likely activity at any given time.

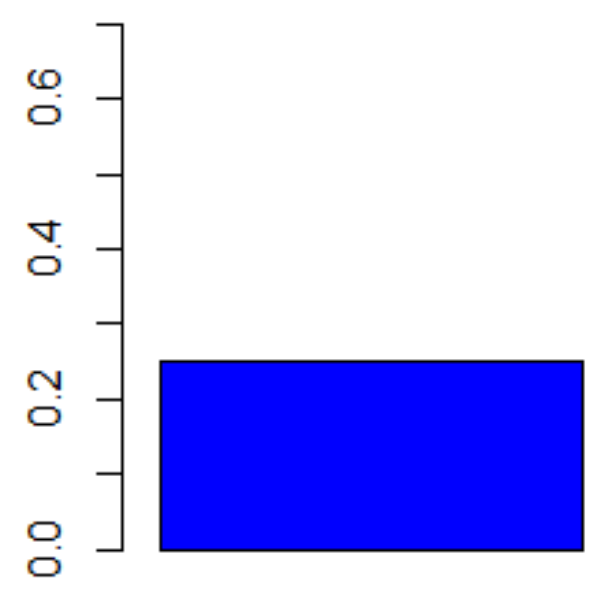

Rainy

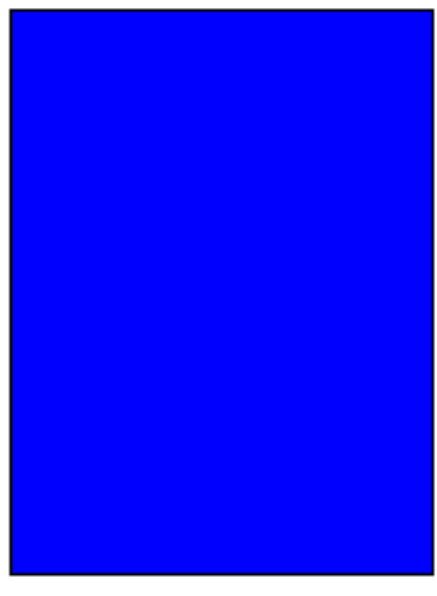

Sunny 


\section{Dry Alluvium Geology (DAG) Test Case Study}

- Case study: Dry Alluvium Geology (DAG) test, an explosive test that was conducted at the Nevada National Security Site.

- Observation data: equipment (cranes, forklifts, etc.) in use at several evenly spaced time points.

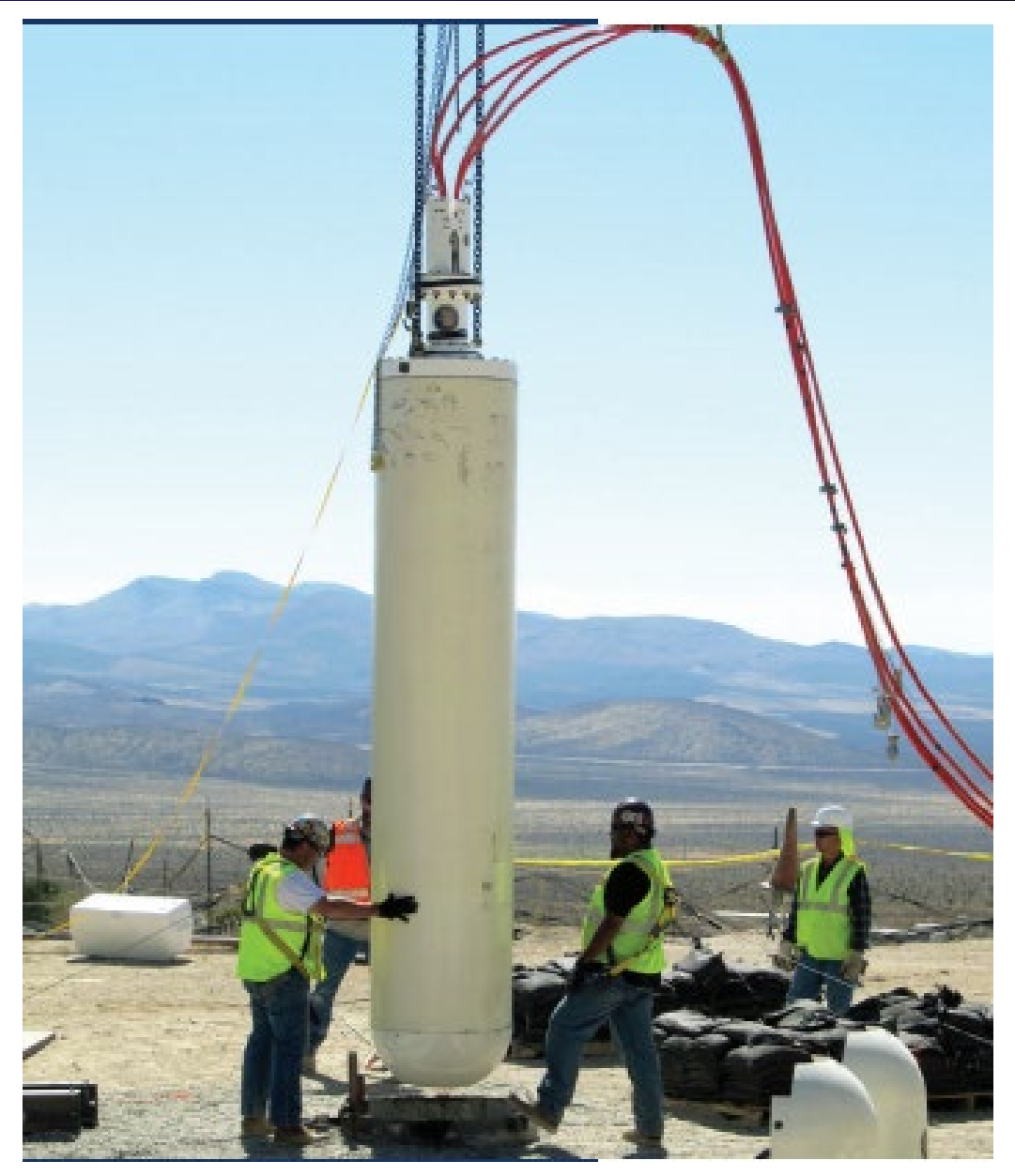




\section{Domain Awareness: Parameterizing DAG HMM}

- How do we incorporate expert knowledge to make our model domain aware?

- Discrete even simulator:

- a process model built by experts

- used to simulate DAG process runs

- use runs to estimate observation probabilities and average activity completion times

- transition probabilities can be derived from the average activity completion times
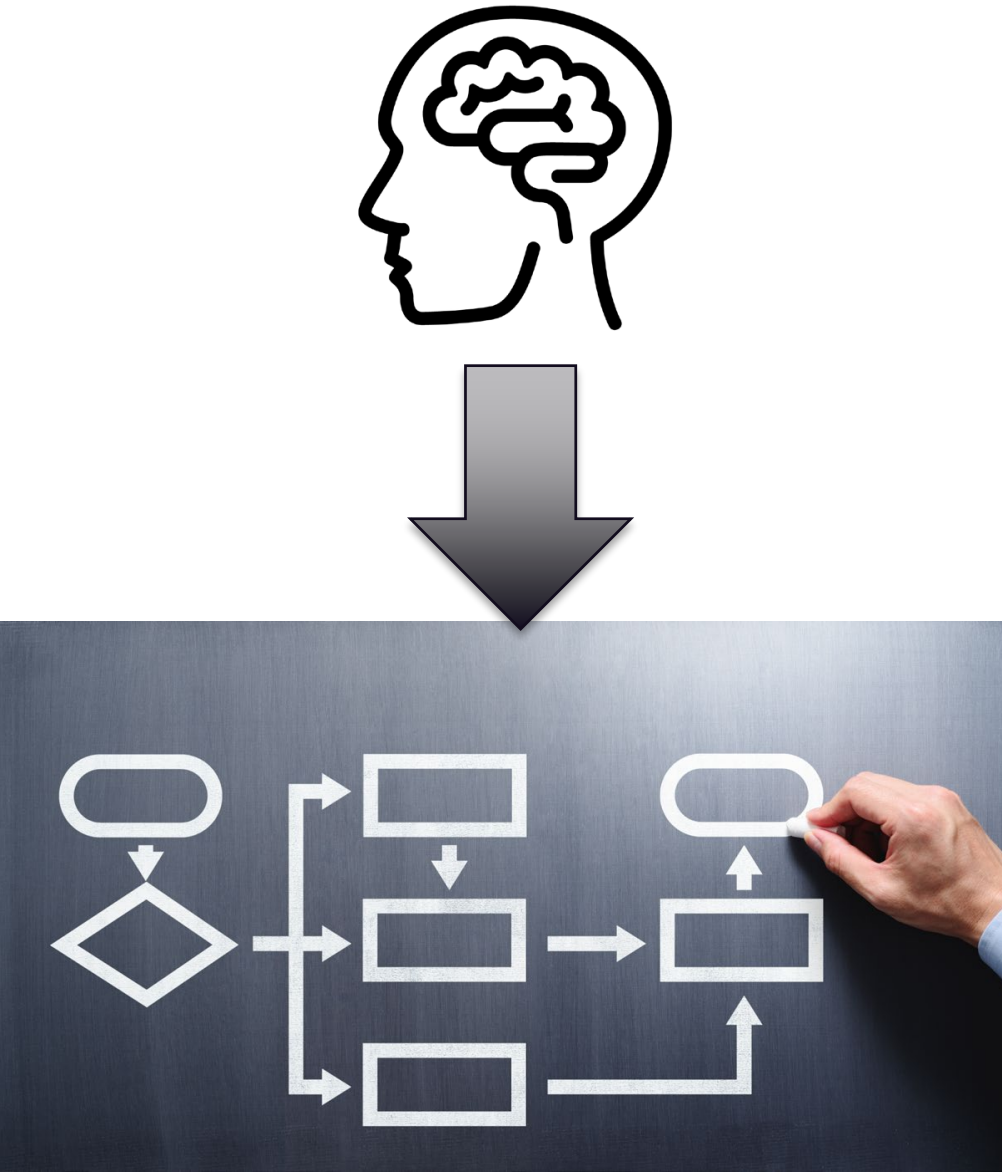


\section{DAG HMM, cont.}

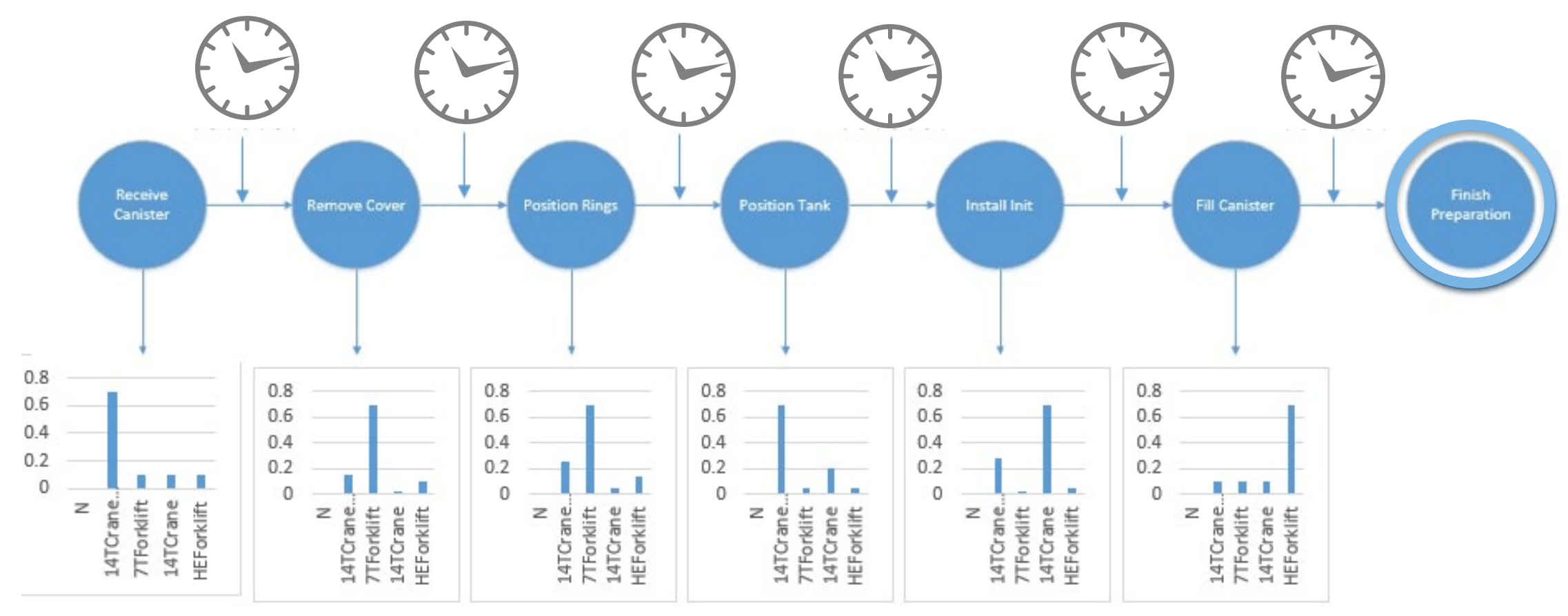




\section{Determining the Most Likely Current Activity}

Initial observation sequence: (14T Crane/Old Glory, 7T Forklift, 7T Forklift, 14T Crane/Old Glory, 14T Crane/Old Glory)
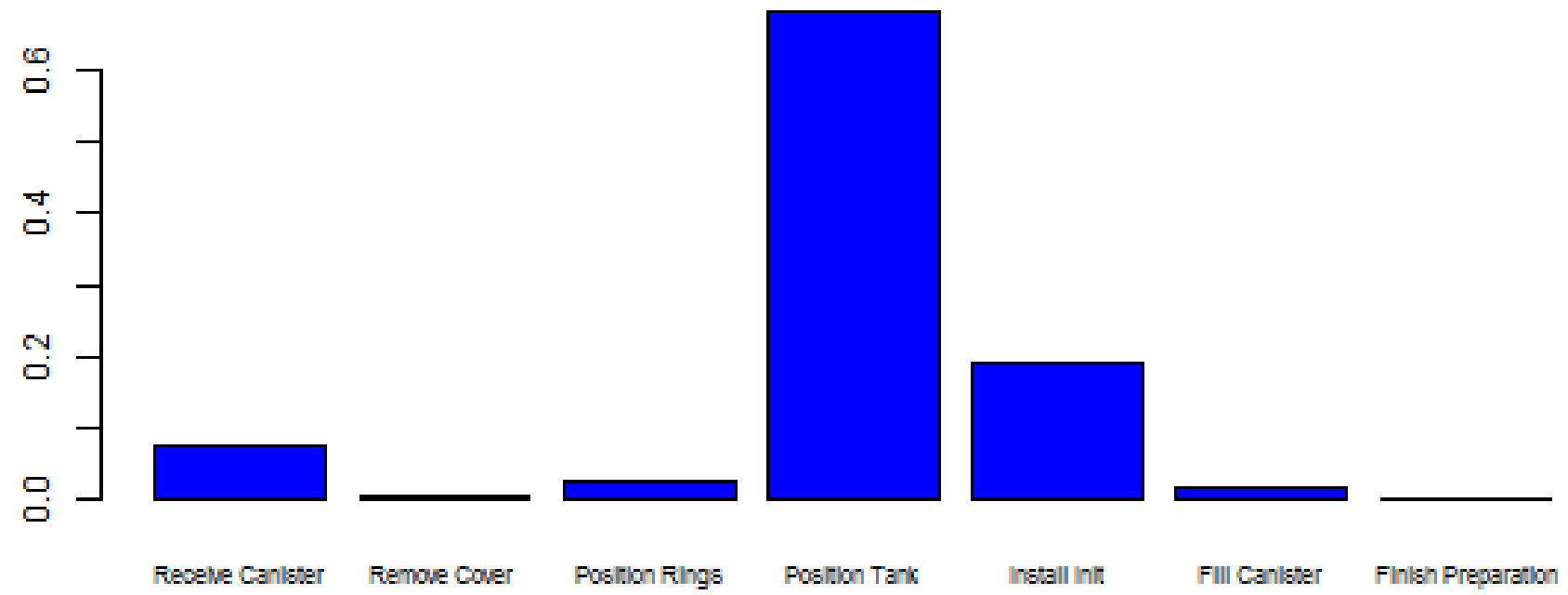

Distribution over process activities after the final observation 


\section{Determining the Most Likely Current Activity (Cont.)}

Replace last two observations with 14T Crane, which is indicative of the "install initiator" activity. Observation Sequence: (14T Crane/Old Glory, 7T Forklift, 7T Forklift, 14T Crane, 14T Crane)
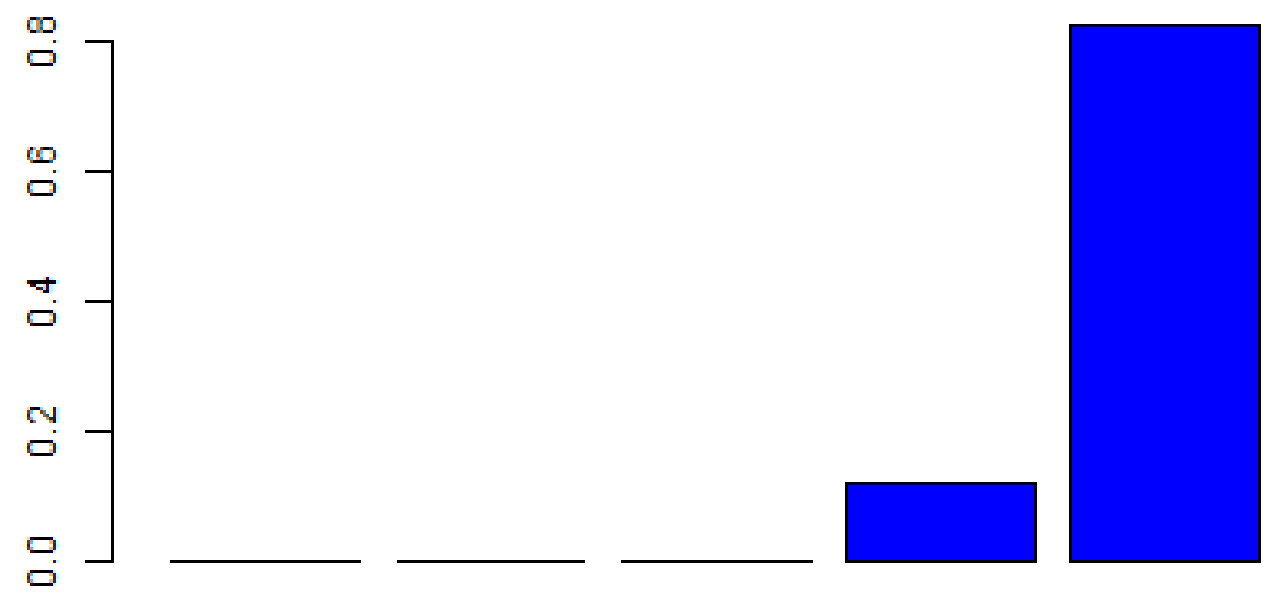

Receve Canlster Fenove Cover Postion Fings Postion Tank Install int

Fill Canlster Finlsh Preparation

Distribution over process activities after the final observation 


\section{Determining the Most Likely Current Activity (Cont.)}

Longer observation sequence with more uncertainty: (14T Crane/Old Glory, 7T Forklift, 7T Forklift, 14T Crane/Old Glory, 14T Crane/Old Glory, 14T Crane/Old Glory, 7T Forklift, 7T Forklift)
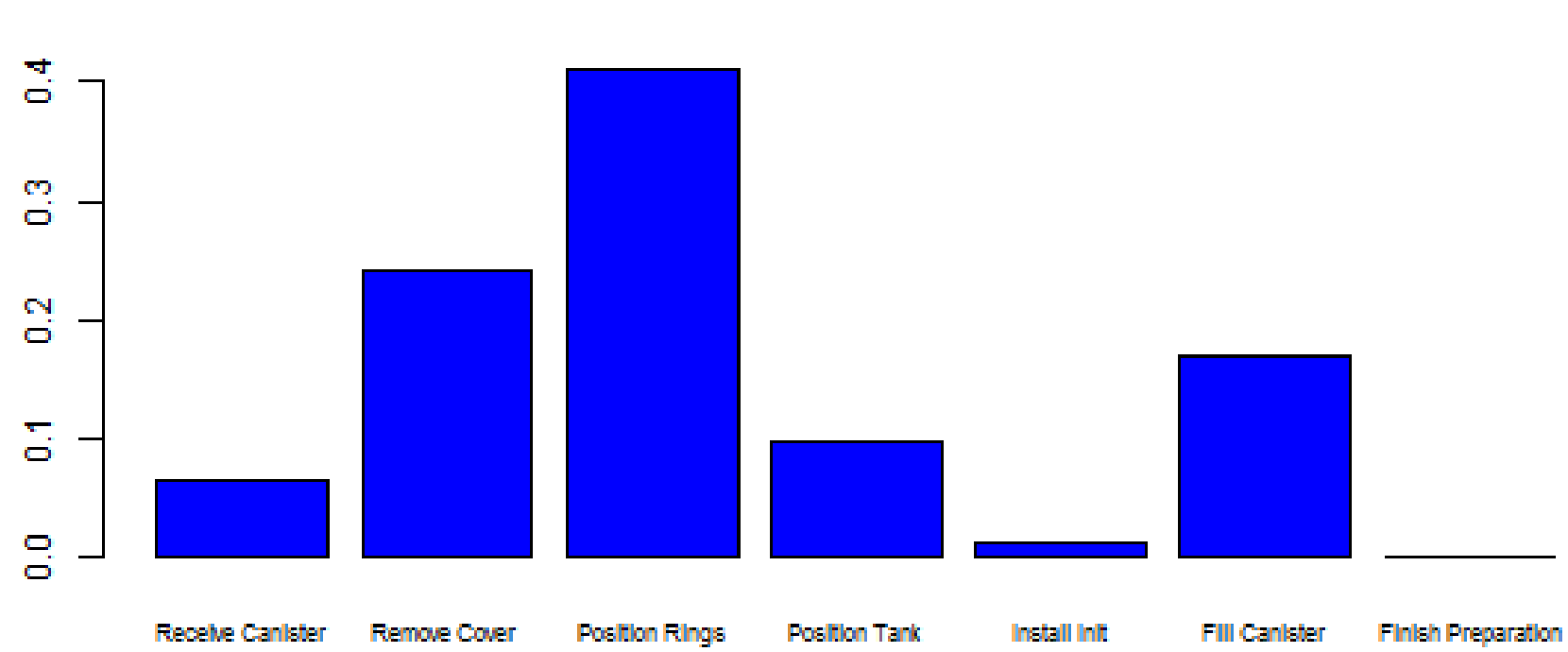

Distribution over process activities after the final observation 


\section{Other HMM capabilities}

\section{The HMM can also}

- determine the most likely sequence of activities corresponding to a sequence of observations

- predict when the process started and when the process will end

- use observed data to update model parameters and quantify parameter uncertainty 


\section{Next Steps}

Next step: Determine what process from a set of processes most likely generated the observed data.

$$
\text { If } \rightarrow \text { Observations }(\boldsymbol{O})
$$

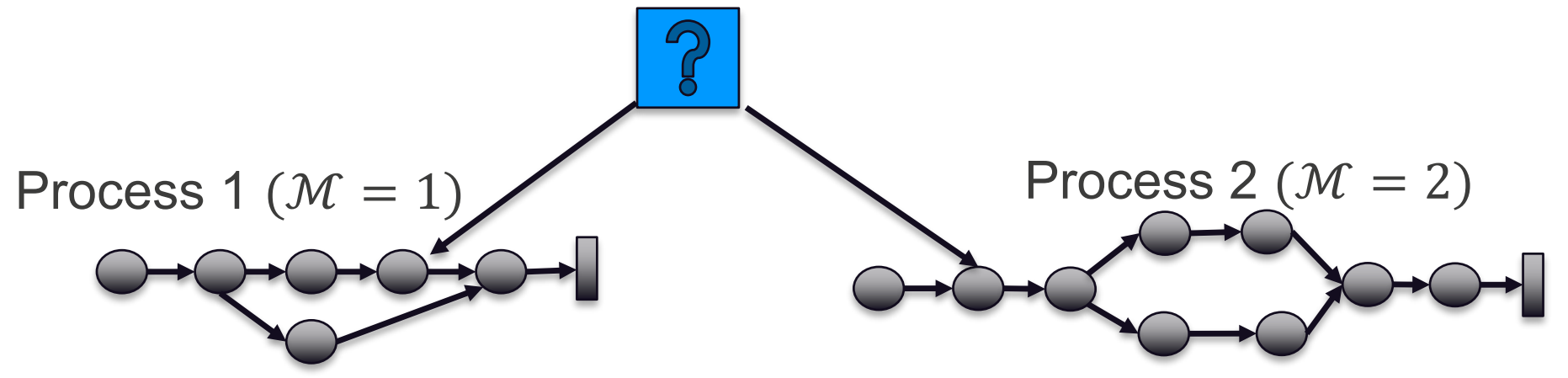

We compute $P(\mathcal{M}=1 \mid \boldsymbol{O})$ and $P(\mathcal{M}=2 \mid \boldsymbol{O})$ and compare. 
Thank you for your attention! 